\title{
Energy Efficient Clustering Protocol to Enhance Performance of Heterogeneous Wireless Sensor Network: EECPEP-HWSN
}

\author{
Santosh V. Purkar ${ }^{1}{ }^{1}$ and R. S. Deshpande (iD $^{2}$ \\ ${ }^{1}$ Department of Electronics and Telecommunication Engineering, Matoshri College of Engineering and Research Center, \\ Nashik Eklahare odhagaon, Affiliated to Savitribai Phule Pune University, Pune, India \\ ${ }^{2}$ S.C.S.M.CO.E, Ahmednagar Nepti, Affiliated to Savitribai Phule Pune University, Pune, India \\ Correspondence should be addressed to Santosh V.Purkar; svpurkar@kkwagh.edu.in and R. S. Deshpande; \\ raj.deshpande@yahoo.co.in
}

Received 1 November 2017; Revised 30 January 2018; Accepted 12 February 2018; Published 22 May 2018

Academic Editor: Youyun $\mathrm{Xu}$

Copyright $\odot 2018$ Santosh V. Purkar and R. S. Deshpande. This is an open access article distributed under the Creative Commons Attribution License, which permits unrestricted use, distribution, and reproduction in any medium, provided the original work is properly cited.

\begin{abstract}
Heterogeneous wireless sensor network (HWSN) fulfills the requirements of researchers in the design of real life application to resolve the issues of unattended problem. But, the main constraint faced by researchers is the energy source available with sensor nodes. To prolong the life of sensor nodes and thus HWSN, it is necessary to design energy efficient operational schemes. One of the most suitable approaches to enhance energy efficiency is the clustering scheme, which enhances the performance parameters of WSN. A novel solution proposed in this article is to design an energy efficient clustering protocol for HWSN, to enhance performance parameters by EECPEP-HWSN. The proposed protocol is designed with three level nodes namely normal, advanced, and super, respectively. In the clustering process, for selection of cluster head we consider different parameters available with sensor nodes at run time that is, initial energy, hop count, and residual energy. This protocol enhances the energy efficiency of HWSN and hence improves energy remaining in the network, stability, lifetime, and hence throughput. It has been found that the proposed protocol outperforms than existing well-known LEACH, DEEC, and SEP with about 188, 150, and 141 percent respectively.
\end{abstract}

\section{Introduction}

Remote event sensing is possible by wireless sensor network, which is composed with certain number of small size sensor nodes with capabilities such as advanced processing, support for communication protocol, transceivers, and sensor with better sensitivity. As wireless sensor nodes or network is easy to deploy and manage, deployment may be uniform, linear, or random based on objectives. To record a real time event, large number of sensor nodes needs to be arrange systematically, such that information collection is possible for low intense and large threshold events for longer span of time. This indicates that the wireless sensor network (WSN) is a cost-effective networking solution for information updating in the coverage radius or in the sensing region. But, the main problem faced by WSN or wireless sensor node is its limited resources. Normally resources available are low storage, less processing power compared to complex instruction set computer, antenna system with limited gain, low energy backup or battery, and low bandwidth for communication. Depending on the application, WSN has its own respective constraints other than low energy backup. Some of the popular applications supported by WSN are in surveillance, forestry, weather forecasting, habitat monitoring, monitoring volcano activity, tracking target in military, machine health updating, inventory systems, and biomedical applications [1-3]. Very different classified applications of WSN are in geoinformatics system (GIS) and intelligent transport system (ITS) [3]. With these examples, applications can be categorized into social, industrial, medical, military, GIS and research field. As WSN is not only composed with wireless sensor nodes, but one of the most important element contributed for WSN is base station (BS) or sink node. To prolong the activity of event monitoring and information updation, it is very necessary to utilize the available energy from the network systematically. Hence, 
there is high demand to design and implement an energy efficient scheme to prolong the performance parameters. Design and implementation of an energy efficient scheme is the commonly suggested solution by researchers in the form of local and global energy saving such as sleep and wake-up strategy, data aggregation, overload reduction, single hop communication, multihop communication, and transmission power control inside WSN. Different approaches proposed by researchers are like data updating at BS through query, threshold, and time based approach [1-4]. Clustering based routing is the most suitable approach to support for load balancing, fault tolerance, and reliable communication and hence to prolong performance parameters of WSN. The clustering scheme has three elements as the cluster head $(\mathrm{CH})$; one of the sensor nodes from respective area works as an intermediate node to transfer concise report of activity from the network in the form of aggregated data to the controller of system that is, BS. Finally, sensor nodes or cluster members (CM) collect and report the event information to $\mathrm{CH}$ regularly or as and when it is required. Indirectly, $\mathrm{CH}$ manages all $\mathrm{CMs}$ locally and utilizes the available resources systematically. $\mathrm{CH}$ also contributes for different responsibility towards the cluster such as channel allocation, intimation of power related information, relay information from lower level to higher level $\mathrm{CH}$ if needed, as well as reforms the cluster and controls the activity of routing based on distance from BS or CM. Finally, clustering scheme is the well-organized routing framework in the sensory system for the sake of energy conservation in WSN. However, clustering scheme provides better energy conservation in WSN, but at run time, there is variation in energy level at sensor nodes of WSN. Hence, network with different energy level nodes is formed. This type of network is referred as heterogeneous WSN and has no longer support to traditional designed clustering protocol, which is based on assumptions of equal energy capability nodes (homogeneous WSN). HWSN is synonymous to real time sensor system, which is composed with different capability nodes for monitoring different parameters. Hence, researchers turn their focus to design and develop clustering protocol for HWSN. Main components of HWSN are same as WSN like sensor nodes and BS, but in this sensor, nodes have different capability such as varied energy backup level, varied antenna system, high processing, and link capability. On the outset, high energy capability nodes is the best choice of all the varied features, as battery energy level controls all the varied features of sensor node. A higher energy backup node is the best choice from the available capabilities, as energy contributes for varied features of sensor nodes. There are the different approaches suggested by researchers to work with clustering scheme as mentioned in $[1,2]$. All those approaches have their respective merits and demerits. In real time, application node whose battery energy level is lower than energy required for sensing accurately or processing is termed as dead node. Thus, energy optimization scheme is also being demanded because it minimizes the death of nodes from WSN. Metaheuristic approach is the preferable optimization scheme to enhance performance parameter. Although heuristic approaches support to extract the full benefit of local energy saving and are greedy in nature for communication establishment, they are found to be less suitable for saving global energy as depicted in [5]. However, the metaheuristic approach is a full proof solution for energy efficiency and finding the best for global. We try to follow the metaheuristic approach for selecting the cluster head for HWSN with three different energy level nodes such as normal, advanced, and super as given in [6, 7]. In the proposed work, we present a distributed clustering approach in the form of hybrid of energy and communication cost. A novel protocol named Energy Efficient Clustering Protocol to Enhance Performance of HWSN (EECPEP-HWSN) is proposed here with different objectives: (i) select the best possible node to be the $\mathrm{CH}$ based on real time information, and (ii) enhance stability period and lifetime by reducing energy consumption in the form of reducing internal overhead and cost of processing energy. In the proposed clustering protocol, there is an assumption of random deployment or distribution of nodes, with the considerations that nodes in remote applications are randomly deployed. All nodes are location aware at the time of deployment. The issue we highlighted here is for the sake of load balancing and minimizing energy consumption of the network by dividing the network initially in four subsections called as zones based on population [8]. All the nodes have to work for the role of $\mathrm{CH}$ or $\mathrm{CM}$ but only one at particular cluster round. A certain amount of clusters and hence the $\mathrm{CH}$ generated inside the network at a time and there location is also known to the other member of network. In the proposed network model, everybody gets an opportunity to work as the $\mathrm{CH}$ or intermediate $\mathrm{CH}$ rather than the role of cluster member. The sensor node or member node plays its role of transferring collected data to respective zone $\mathrm{CH}$, which is in the coverage range. It is well assured that the selected temporary $\mathrm{CH}$ and final $\mathrm{CH}$ are less likely in the or on the vicinity of network.

The proposed work is presented here in the following subsections as second section presents literature survey based on published literature and follow the energy consumption model from the same. Third section offers network details acquired for the proposed work in the form of network model, proposed work in the form of explanation of mathematical base is presented in the form of proposed protocol in fourth section, and proposed work simulation and performance parameters are explained in fifth section. Simulated work and its validation with existing well-known protocol is elaborated in section six, and final outcome of proposed work is presented in the form of conclusion with future scope in last section.

\section{Literature Survey}

Though the design and implementation of energy efficient clustering protocol are demanded, there are number of issues raised during real time working. Designing an energy efficient routing based on the cluster scheme to support heterogeneity, scalability, and hierarchy in topology are the main goals of researchers in the area of wireless sensor network to prolong the lifetime [1, 2]. Energy efficient clustering is possible by initiating load balancing among the 
nodes and must keep scheme excursion free in terms of energy. To prolong the lifetime of WSN or heterogeneous WSN, researchers put a lot of effort to design energy-efficient clustering scheme with a varied approach than clustering with hierarchical topology as presented in [3]. In this protocol, $\mathrm{CH}$ selection is based on calculating routing energy cost required by node with reference to BS from the respective group. Authors used the Gaussian elimination method to select the most feasible low-loss route with reference to node for the role of $\mathrm{CH}$. The second point is that the $\mathrm{CH}$ node near to $\mathrm{BS}$ is selected as the first-level $\mathrm{CH}$ and $\mathrm{CH}$ node at longer distance as lower level $\mathrm{CH}$. This offers the best suitable route discovery scheme to avoid loossy path (the path which have weak or lower energy nodes) and hence enhance energy efficiency, improves stability, and lifetime of the network. But this scheme has less consideration of selected $\mathrm{CH}$ position and less curious about energy consumption rate adopted by particular node. Hence there is high possibility of abrupt breakage in stability and hence reduced lifetime of the network. This is one of the best examples of multihop routing but it also possesses the limitations of multihop routing. In scalable environment, routing table was found to be huge and it imposed higher energy load on the respective node. To achieve greater energy efficiency and to reduce internal overhead, a different approach of dividing the available network into multiple clusters is proposed by Rana and Zaveri in the form of [9].

In this scheme, less loss energy path is selected with reference to nodes for the role of $\mathrm{CH}$. Updation of energy information is needed regularly for selecting best suitable routing path, but it imposes extra load in case of scalable network. Sometimes the $\mathrm{CH}$ node may be at longer distance from BS, which increases energy consumption. To support load balancing and efficient resources, utilization in the form of single hop cluster routing for homogeneous WSN is initiated by Heinzelman et al. [10]. This is one of best protocols to support the objective of load balancing by providing chance to each and every node to become the $\mathrm{CH}$ over a life of network. But, the node which is elected as $\mathrm{CH}$ for particular round would never be $\mathrm{CH}$ again in the next $1 / p$ round with probability of cluster head $p$. CH election is based on random number generation by nodes within the range of 0 to 1 . The node whose values are less than the threshold $T(n)$ has been elected as cluster head for particular round. Once the node is elected as $\mathrm{CH}$, then it publishes its identity and associated parameter in the form of advertise ADV message to the CM in the coverage zone. The CM replied to ADV with associated parameters like time slot acquired, energy level available, hop count, and so on. Based on the reply received from the CM nodes of its coverage, the $\mathrm{CH}$ prepares TDMA and publishes it on air. With this, everybody comes to know the time slot on which to participate for communication. Other than this slot period, a particular node can be in sleep state, to save energy. A merit from this work is that the $\mathrm{CH}$ role is rotated among available sensor nodes such that load balancing is achieved. With single-hop approach, considerable energy saving is possible. But, there are a higher number of demerits to compensate for merits as no basis of residual energy for the selection of $\mathrm{CH}$ and rotation of $\mathrm{CH}$ impose possibility that the node elected as the $\mathrm{CH}$ is on the vicinity of network or in the vicinity. Lastly, as basis of centralized clustering approach is utilized for design, it is less suitable for scalable network. Minimizing this problem by the support of optimum number of cluster and using available energy faithfully is initiated by Wang et al. [11]. This protocol works on the principle of LEACH as per random approach, but with the consideration of residual energy and death toll calculation from BS. Decision based on the available time period before the complete energy depletion helps in $\mathrm{CH}$ selection. This improves the stability period and hence the lifetime of the network. This approach enhances the energy utilization with sacrifice of increased internal overheads, which requires high processing energy and impose load on network performance.

Extension of Low Energy Adaptive Clustering Hierarchy $(\mathrm{LEACH})$ work is presented by $\mathrm{Li}$ and Liu [12]. The main objective of this work is to design an energy efficient clustering protocol to improve energy remaining in the network and prolong the lifetime of the network. This is to compensate for the assumptions of all equal energy nodes available inside the network during different clustering cycles. Hence, this protocol considers residual energy of the node and distance with respect to BS, and the other cluster member is considered for the selection of $\mathrm{CH}$. The $\mathrm{CH}$ node with lower distance with respect to BS is treated as second level $\mathrm{CH}$ and the node with higher distance as level-1 $\mathrm{CH}$. In this level-1, the $\mathrm{CH}$ transfers collected data on behalf of cluster to level-2 for further communication to BS. Hence the burden of communication (transmission) is reduced for level-1 $\mathrm{CH}$ and considerable energy saving in case of level-2 is achieved. Hence, energy utilization is systematic. But, to continue for next cycle, parameters need to be updated at the end of every cluster cycle. In this case, also $\mathrm{CH}$ selection threshold probability for both levels is different from normal LEACH, which results in energy excursion between two different $\mathrm{CH}$ selections. But, this scheme was unable to incorporate initial energy value in $\mathrm{CH}$ selection process. This scheme is less supportive in case of different capability nodes. To resolve the said issue, a solution in the form of Hybrid, Energy-Efficient, Distributed Clustering (HEED) protocol is proposed by Younis and Fahmy [13]. In this scheme, $\mathrm{CH}$ selection is based on node degree and residual energy with one addition parameter in the form of relative distance. The selected $\mathrm{CH}$ was found to be more faithful than earlier explained protocol. But, the selected node is less assured in network premises and hence results in higher energy dissipation. This leads to stability breakage, and hence lifetime is affected badly; designed protocol is not suitable for heterogeneous WSN and unable to explore average energy utilization.

Stable Elections Protocol (SEP) is introduced to enhance stability and lifetime of heterogeneous WSN by Smaragdakis et al. [14]. Two level energy nodes are introduced in this protocol namely, normal and advanced nodes. Basically in HWSN, nodes those are heterogenite in resources are less in percentage fraction. In this scheme, $\mathrm{CH}$ selection is based on available initial energy with nodes. Normally, the advanced node has higher energy level backup than its follower node. The probability of node to be the $\mathrm{CH}$ is different for both 
types of node, but mostly the advanced node has more chance to get selected as $\mathrm{CH}$ than normal node. In the study, increasing percentage factor of advanced nodes and hence probability of $\mathrm{CH}$ selection improves performance in the form of stability and lifetime, which also improves throughput of the network. Demerits of the said system are advanced node get punished badly as there is no reference of distance between selected $\mathrm{CH}$ node and BS, they have more chance to get selected as $\mathrm{CH}$ and hence reduce the network survival time of network. To reduce the possibility of selecting mostly one type of node (e.g.,advanced nodes) for the role of $\mathrm{CH}$, a new approach called Distributed Energy- Efficient Clustering (DEEC) protocol is proposed by Qing et al. in [15]. Distributed energy efficient clustering (DEEC) is another HWSN clustering protocol having different approach of selecting $\mathrm{CH}$. In DEEC, $\mathrm{CH}$ selection is based on ratio of residual energy to average energy of the network. By varying the epoch period, we can have a well-balanced scheme of $\mathrm{CH}$ selection for different types of node. The node whose energy level, initial or residual energy, is higher gets more chance to become $\mathrm{CH}$ than the node with low energy level. But, DEEC have the constraints as network lifetime is recorded and helping in computing reference energy available for further activity. DEEC have the same problem as SEP protocol as to punish advanced node badly and need to kept global knowledge of network. Elbhiri et al. presents a different approach from DEEC in the form of Developed Distributed Energy-Efficient Clustering protocol in [16]. In the study, they uses initial and residual energy to select $\mathrm{CH}$. But, for the said selection, all the nodes must have global knowledge of the network. This scheme also uses the concept of computing reference energy for cluster round. This protocol has different probability of $\mathrm{CH}$ selection for different types of nodes. To resolve this up to certain level author of Enhanced Developed Distributed Energy-Efficient Clustering (EDDEEC) suggest $\mathrm{CH}$ selection criteria based on residual energy and average energy of the network, and change the probability of $\mathrm{CH}$ selection for different type of node [17]. As this protocol accounts for three different types of nodes, probability of $\mathrm{CH}$ selection is based on respective weights. This nonprobabilistic approach gives less importance to relative and average distance in selecting the $\mathrm{CH}$ node. This scheme also has lower association with other members; this can be improved by adding distance parameter in $\mathrm{CH}$ selection.

Other approach based on LEACH for HWSN with three different kinds of nodes is proposed by Kumar et al. [18]. In the study, $\mathrm{CH}$ selection is based on residual energy and relative distance in hierarchical clustering network. With hierarchical network, multilevel clusters are formed in case of scalable network. This creates unequal size of clusters inside the network. Hence, it affects the performance parameters supported by the protocol. Large numbers of clusters affect network survival time and hence reduce the stability and lifetime from the network.

Finally, one of the different approaches presented by author for exclusive network arrangement is given in [19]. In the study, clustering of multiple profile nodes are presented by the author and their structure topology is proposed in the form of multiple cluster. Nodes from different clusters communicate differently and form a virtual network of same type of nodes to initiate communication of event data updation. In this, nodes with smallest distance and better node degree will be selected as $\mathrm{CH}$. But, selected $\mathrm{CH}$ may be at the vicinity and creates unnecessary energy dissipation. Hence, stability and lifetime get affected badly. A novel scheme of energy-efficient event driven hybrid routing protocol proposed by author in [20]. A main objective of this scheme is to distribute available heavy traffic evenly over the clustered network by offering unequal size cluster environment. Selected $\mathrm{CH}$ offers better node degree and hence achieves better energy efficiency. Cluster near the sink is normally of smaller size than moderate size cluster in the middle of the network. The approach of $\mathrm{CH}$ selection is based on available energy on routing path, nodes with higher energy on path selected as the best suitable node for the role of $\mathrm{CH}$. This scheme enhances energy efficiency of the network and hence improves lifetime, but less assurance for selected node in the premises of network. Follow the strategy of multihop communication for information transfer. To enhance energy efficiency in HWSN with the feature of scalability taken into account, a new protocol was proposed by author in [21]. In this scheme, original hybrid energy efficient distributed (HEED) work is explored through the base of fuzzy logic with three different types of nodes. $\mathrm{CH}$ selection is based on node density, residual energy, and the distance between node and sink. Fuzzy logic is used to calculate the most probable node to be $\mathrm{CH}$ for further activity of network. About 27 different combinations are evaluated for best suitable node selection. But, again control heads for network management are higher and consume greater energy for $\mathrm{CH}$ calculation. Probability function value changed for every iteration imposes burden on network energy. Though there are multiple schemes available to prolong performance parameters of HWSN, all those are somehow are less faithful to really enhance the energy efficiency of WSN without considering their $\mathrm{CH}$ selection criteria and network model support.

Hence to design and implement energy efficient clustering with heterogeneous WSN, a novel approach of $\mathrm{CH}$ selection is proposed in this article such that energy utilization of the network is improved. Hence, all respective performance parameters get improved.

\subsection{Motivation. There are certain issues found in the above-} mentioned clustering protocols in terms of faithful $\mathrm{CH}$ selection, energy-efficient cluster formation, and network management. There is the $\mathrm{CH}$ selection criteria indirectly linked with probabilistic approach or connected to threshold $T(n)$ with probabilistic approach. By varying the $\mathrm{CH}$ selection criteria it is possible to select most promising node to be $\mathrm{CH}$, but it increases internal overhead. Sometimes, the node selected as $\mathrm{CH}$ is at the vicinity or in the vicinity, which increases the energy consumption in the network and hence reduces the performance of network. So, the node with this position is not suitable for the role of $\mathrm{CH}$. Hence, the nodes must have better internal parameter 
and better connectivity with nodes and mainly situated in the premises of network. Hence, a better $\mathrm{CH}$ selection approach enhances the performance parameters of the network.

2.2. Energy Consumption Model. Communication section consists of transmitter and receiver; transmitter section of sensor nodes consumes energy to run radio electronics and amplifier circuit. On the contrary, the receiver section consumes energy only in the receiving packet at radio electronics over communicating distance [5, 14]. If the communication entities separated by distance $d$ is less than a threshold distance, then free space energy consumption model is used for energy expenditure calculation else multipath model is utilized. Data aggregation feature is incorporated for $\mathrm{CH}$ to $\mathrm{BS}$ delivery is same as $[5,14]$. While transmitting a packet of length $L$ to a distance $d$, energy expenditure is calculations are presented in (1).

Here, parameters involved have their respective significance as $E_{\text {elec }}$ is the energy consumed by transmitting or receiving electronics circuitry to process single bit information. $E_{\mathrm{tx}}$ and $E_{\mathrm{rx}}$ are the energies consumed by transmitting and receiving section to process data packet of length $L$, which also depend on digital coding and on digital modulation scheme utilized. $E_{\mathrm{fs}}$ and $E_{\mathrm{mp}}$ are the amount of transmitter amplifier expenses in the form of energy required for free space model (free space energy loss) and for multipath model, respectively. Main classified information about amplifier (power amplifier) is to control the setting of power such that if communication distance $d$ between sender and receiver is less than $d_{0}$, then free space power loss model is to be considered for energy calculation.

The energy required at transmitter side with free space model is

$$
E_{\mathrm{tx}}(L, d)=L E_{\mathrm{elec}}+L E_{\mathrm{fs}} d^{2} \quad d \ll d_{0} .
$$

If the distance $d$ is greater than threshold distance, then multipath model is utilized for energy expenses calculation as

$$
E_{\mathrm{tx}}(L, d)=L E_{\mathrm{elec}}+L E_{\mathrm{mp}} d^{4} \quad d \gg d_{0} .
$$

Energy consumption at receiver side is

$$
E_{\mathrm{rx}}(L, d)=L E_{\text {elec }},
$$

where $L$ is the length of data packet in number of bit for communication. Calculation of $d_{0}$ is

$$
d_{0}=\frac{\sqrt{E_{\mathrm{fs}}}}{\sqrt{E_{\mathrm{mp}}}} .
$$

\section{Network Model}

In the proposed network model, we use three-level node energy heterogeneity. The network model consists of $n$ randomly deployed sensor nodes on $M \times M$ sensing layout. It is predefined as all the nodes including BS are static on postdeployment. Communication links between each other are assumed to be symmetric [14]. The $\mathrm{CH}$ on behalf of sensing network is responsible to forward collected data directly to BS. All supervisory and unsupervisory data messages are transacted through wireless links.

Initially, network layout is divided in four zones based on population, and BS is at the center of network field. Every node has the capability to sense, aggregate, and forward the data. It is a prime consideration that the node always have data for transfer. Nodes namely normal, advanced, and super with increasing order of energy level as presented in [14]. For our simulation, we are using energy increment factor of about twice the former. Hence, in the proposed protocol, we have three-level energy value of heterogeneity as $E_{0}, E_{\mathrm{Adv}}$, and $E_{\text {Super }}$ for energy of normal, advanced, and super nodes, respectively. Nodes with percentage population factor as $a$ for advanced nodes with total $n$ nodes, which is equipped with energy factor greater than $m$ times than normal node. Super nodes are equipped with energy incrementing factor as $m_{0}$, with percentage population factor $a_{0}$ with respect to total $n$ nodes. Individual node details in the form of equation are presented as follows.

Initial energy for normal nodes is $E_{0}$ with population count as $n\left(1-a-a_{0}\right)$. Subsequently, advanced and super node populations are $n a$ and $n a_{0}$, respectively. Energy available with advanced and super node is $E_{\mathrm{Adv}}$ and $E_{\text {Super }}$, respectively.

Energy calculations for respective nodes are as follows: Energy contributed by the normal node is

$$
E_{\text {normal }}=n E_{0}\left(1-a-a_{0}\right) \text {. }
$$

Energy by the advanced node is

$$
E_{\mathrm{Adv}}=n E_{0}(1+m) a \text {. }
$$

Energy due to the super node is

$$
E_{\text {Super }}=n E_{0}\left(1+m_{0}\right) a_{0} \text {. }
$$

Equations (5-7) present energy available with all three types of nodes. Total initial energy proposed in the network model is calculated as $E_{\mathrm{Tot}}$ :

$$
\begin{aligned}
E_{\mathrm{Tot}}= & E_{\text {normal }}+E_{\mathrm{Adv}}+E_{\text {Super }}, \\
E_{\mathrm{Tot}}= & n E_{0}-n a E_{0}-n a_{0} E_{0}+n a E_{0}+n E_{0} m a \\
& +n a_{0} E_{0}+n a_{0} m_{0} E_{0} .
\end{aligned}
$$

In (9), it is found that the second and fourth terms are of equal magnitude and are out of phase. Terms third and sixth are of the same magnitude and are out of phase. Hence, (9) is rewritten as

$$
E_{\mathrm{Tot}}=n E_{0}+n E_{0} m a+n a_{0} m_{0} E_{0}=n E_{0}\left(1+m a+m_{0} a_{0}\right) .
$$

Hence, from (10), we hereby conclude that if we add heterogeneity level up to level-2, available energy increased by factor $(1+m a)$, and if it is increased to level 3 , then energy increased by factor $\left(1+m a+m_{0} a_{0}\right)$. If we would like to improve heterogeneity to " $N$ " level greater than 2 , then the equation is modified as given below: 


$$
E_{\mathrm{Tot}}=n E_{0}\left(1+m a+\sum_{i=0}^{N}\left(m_{i} a_{i}\right)\right), \quad i=0, \ldots, N
$$

where $N$ can be any integer number.

It means that the network with $N$ level heterogeneity has energy level $\left(1+m a+\sum_{i=0}^{N}\left(m_{i} a_{i}\right)\right)$ times greater than normal nodes involved in network (Homogeneous Wireless Sensor Network), but the constraints of node energy heterogeneity level is greater than 2 .

In the proposed scheme, the $\mathrm{CH}$ selection approach is nonprobabilistic rather than probabilistic. We can very well calculate other supporting parameters involved in clustering processes as follows.

Number of rounds supported by the network before complete death can be calculated as

$$
R=\frac{E_{\text {Tot }}}{E_{\text {Round }}},
$$

where $R$ is denoted as total number of rounds supported by network before death of all nodes. $E_{\text {Round }}$ is the energy dissipated by the network for completing one round and can be calculated as

$$
E_{\text {Round }}=K\left(2 n E_{\text {elec }}+n E_{\mathrm{DA}}+L E_{\mathrm{mp}} d_{\mathrm{to} \mathrm{BS}}^{4}+n E_{\mathrm{fs}} d_{\mathrm{to} \mathrm{CH}}^{2}\right),
$$

where $K$ is the optimal number of clusters generated, $E_{\mathrm{DA}}$ is the data aggregation energy for $\mathrm{CH}, d_{\text {to BS }}$ is the distance between $\mathrm{CH}$ to $\mathrm{BS}$, and $d_{\text {to } \mathrm{CH}}$ is the distance between cluster members to $\mathrm{CH}$.

$$
\begin{gathered}
d_{\text {to BS }} \text { and } d_{\text {to } \mathrm{CH}} \text { are calculated as } \\
d_{\text {to } \mathrm{CH}}=\frac{M}{\sqrt{2 \pi K}}, \\
d_{\text {to BS }}=0.765 \frac{M}{2} .
\end{gathered}
$$

Taking the derivative of $E_{\text {Round }}$ with respect to $k$ and equating it zero gives optimum number of cluster heads $K_{\text {opt }}$ and is calculated as

$$
\begin{aligned}
& K_{\mathrm{opt}}=\frac{\sqrt{n}}{\sqrt{2 \pi}} \times \frac{\sqrt{E_{\mathrm{fs}}}}{\sqrt{E_{\mathrm{mp}}}} \times \frac{M}{d_{\mathrm{to} \mathrm{BS}}^{2}}, \\
& P_{\mathrm{opt}}=\frac{K_{\mathrm{opt}}}{n},
\end{aligned}
$$

where $P_{\text {opt }}=0.1$ is the optimum probability of number of clusters.

\section{Proposed Protocol}

Main objectives of proposed energy efficient clustering protocol are to reduce internal overheads of network management, reduce the possibility of selected $\mathrm{CH}$ on the vicinity, and support scalability. Select the node for the role of $\mathrm{CH}$ such that the $\mathrm{CM}$ is able to communicate to BS in a fair way, without introducing extra load on other nodes. Selection of $\mathrm{CH}$ must be with run time parameter. At the end of every cluster cycle, all nodes update their parameter information to BS. Design clustering protocol must support scalability features. With all these objectives, we are presenting here a novel scheme of energy efficient clustering protocol to enhance performance metrics of HWSN. Initially, all the nodes are deployed randomly in equal percentage over the network; the network is divided into four sections based on population density in the form of zone $A$, Zone $B$, zone $C$, and zone $D$ as given in [8].

$$
\text { Total network area }=\text { area }(A+B+C+D) .
$$

This scheme reduces the internal overhead for network management; this improves the energy utilization and enhances energy remaining in the network at run time. Some of the issues of existing clustering protocol are such as cluster size is arbitrary, selected $\mathrm{CH}$ is located towards the boundary of the network and hence energy depletes at faster rate. This affects the network survival time and network performance. In the proposed work, we are selecting the node for the role of $\mathrm{CH}$ is based on real time parameter available with the sensor node. We introduce a very new profile parameter named as Node Quality Index or Node Index (NI), which is a fusion of node real-time parameters. Node Quality Index is the devised parameter based on initial energy, residual energy, and the hop count required by particular node with respect to base station. As for $\mathrm{CH}$ selection based on energy, there are some of the criteria such as initial energy with node must be high enough to handle the role of $\mathrm{CH}$ for cluster management activity such as advertisement, frame formation, and data packet transaction with respect to BS (energy greater than or equal to this is expected), after cluster round, energy remaining with the node must be high enough to handle the responsibility of sensor node (higher energy level is expected such that node can be $\mathrm{CH}$ in upcoming rounds), and the assurance of aliveness and finally node must be moderately or nearly situated from the base station. The selected $\mathrm{CH}$ will be able to support scalable network for multihop communication. Mathematical representation of the proposed scheme is presented in the form of mathematical model given below.

4.1. Protocol Explanation. In this proposed work, we are presenting a new approach of $\mathrm{CH}$ selection by introducing a very new parameter named as Node Quality Index $\left(Q_{n i}\right)$. Node Quality Index is the fusion of initial energy of the node, current available energy at run time, and hop count with respect to base station. For devising node index, we are using index modelling from the available different modelling approaches of database system.

Before the execution of the above-mentioned steps, one of the important phases needs to execute for faithful execution of complete algorithm as for some clustering cycle, and it is found that most of the nodes have equal value of NI. So filtering phenomenon is found to be complex, and to minimize the complexity, we incorporate LEACH policy for selecting $\mathrm{CH}$ for small number of rounds (e.g., 50 rounds). With this approach, we initially have the approach of load 
Prerequisites:

(i) All nodes are location aware and updated with neighbor information.

(ii) Nodes always have data ready to transfer at every cluster cycle.

(1) Start

(2) Nodes from each zone send their respective attributes to BS

(3) BS collect and update it in database

(4) BS calculate NI for each node for every zone

(5) BS list number of nodes with NI

(6) Calculate the average value of NI

(7) BS compare calculated NI with Average NI

(8) Number of nodes whose value greater than average NI are listed

(9) Nodes those are about $\pm 10 \%$ of boundary are sorted zone wise

(10) Nodes those are on the verge are filtered if other member is available from the zone

(11) Intimate nodes from each zone as selected temporary $\mathrm{CH}$ for that zone

(12) Node whose value greater than average NI of competitive nodes selected as Final CH on behalf of network

(13) Temporary $\mathrm{CH}$ updated with final $\mathrm{CH}$

(14) Before transferring data packet to $\mathrm{BS}$, temporary $\mathrm{CH}$ has to check distance with respect to $\mathrm{BS}$ and final $\mathrm{CH}$

(15) If distance between final $\mathrm{CH}$ is greater than BS, then send collected data packet to BS directly

(16) Final $\mathrm{CH}$ send only collected data packets from others to BS

(17) Again back to step 2.

\section{Algorithm 1}

balancing; with initial network division, we achieved improved energy utilization (in the form of reduced internal overheads), and the size of formed cluster is controllable than the arbitrary size of network [8]. We target the applications with proposed protocol are such as in surveillances, weather forecasting, home automation, traffic management, habitat monitoring, in machine health analysis, and inventory management.

The fundamental $\mathrm{CH}$ solution applied for proposed work is

$$
\mathrm{CH}_{i}= \begin{cases}Q_{\mathrm{ni}}>\mathrm{Avg}_{i}, & \text { for } i>50 \\ Q_{\mathrm{ni}} \frac{P_{i}}{1-P_{i}(\mathrm{rmod})\left(1 / P_{i}\right)} & \text { for } i=0 \text { to 50, }\end{cases}
$$

where $\operatorname{Avg}_{i}=\left(\sum_{i=0}^{n} Q_{\mathrm{ni}} /\left|Q_{\mathrm{ni}}\right|\right)$.

Boundary $=$ boundary of network layout $\pm 10 \%$.

$$
\mathrm{CH}_{i}=\left[\text { boundary } \cap\left(Q_{\mathrm{ni}}>\operatorname{Avg}_{i}\right)\right] \text {. }
$$

\section{Simulation Parameters}

This section highlights the parameters set for implementation and performance validation for simulation work. Some of those are explained as follows:

(i) Stability period: The time period before the death of very first node from available sensor nodes of operating WSN (HWSN).

(ii) Number of alive nodes per cluster round: Number of nodes alive from the network for every cluster round, which indirectly presents the available energy remaining in the network.

(iii) Number of dead nodes per cluster round: Number of nodes dead per cluster round against changing energy level inside the network during network survival time. This factor indirectly presents death rate of nodes over cluster cycle. Which indicate possible lifetime remains with network in the form of cluster round.

(iv) Throughput: Number of data packets sent from the sensor nodes towards base station over the cluster round presents amount of throughput per cluster round. Amount of throughput signifies energy efficient utilization of available network resources. Throughput presents quality of the network.

Parameter set utilized for the purpose of simulation is presented in the form of Table 1.

\section{Discussion}

This section focuses on implementation strategy and results of our simulated work and its validation with well-known published protocol such as LEACH, SEP, and DEEC.

With reference to parameter set given in Table 1 , we randomly deploy the network in $200 \mathrm{~m} \times 200 \mathrm{~m}$ form. Divide the network into four zones based on population; placement of BS is at the center of the network with position $(100 \times 100)$. Total node population over the network is 200, with percentage fraction factor $a=0.2$ and $a_{0}=0.1$. With advanced node energy value about to double the normal node energy, super node energy value is also about twice of advanced node.

6.1. Validation with LEACH Protocol. As we know LEACH strategy is best suitable for improving lifetime of WSN by offering an equal opportunity to everybody to become a $\mathrm{CH}$ and balance the energy consumption as proposed in [10]. But it is a random selection of $\mathrm{CH}$; there is no reference of residual energy as well as hop count of selected $\mathrm{CH}$ with reference to 
TABLE 1: Simulation parameter.

\begin{tabular}{lccc}
\hline Serial Number & Parameter symbol & Name & Value \\
\hline 1 & $M \times M$ & Network area & $200 \mathrm{~m} \times 200 \mathrm{~m}$ \\
2 & $N$ & Number of nodes & 200 \\
3 & $E_{0}$ & Initial energy of nodes & $0.5-1.5 \mathrm{~J}$ \\
4 & $L$ & Data packet size & $4000 \mathrm{bits}$ \\
5 & $E_{\text {elec }}$ & Radio electronics energy & $50 \mathrm{~nJ} / \mathrm{bit}$ \\
6 & $E_{\varepsilon f s}$ & Free space energy & $10 \mathrm{pJ} / \mathrm{bit} / \mathrm{m}^{2}$ \\
7 & $E_{\mathrm{mp}}$ & Amplification energy & $0.0013 \mathrm{pJ} / \mathrm{bit} / \mathrm{m}^{4}$ \\
8 & $E_{\mathrm{DA}}$ & Data aggregation energy & $5 \mathrm{~nJ} / \mathrm{bit} / \mathrm{signal}$ \\
9 & $d_{0}$ & Threshold distance & $87-87.7 \mathrm{~m}$ \\
10 & $\mathrm{BS}$ & Sink node & $(100,100)$ \\
\hline
\end{tabular}

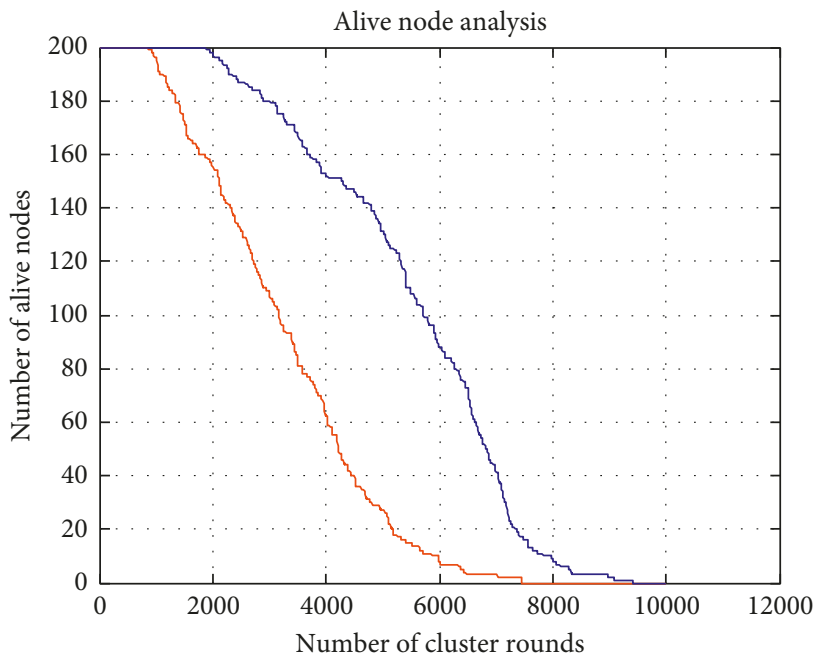

(a)

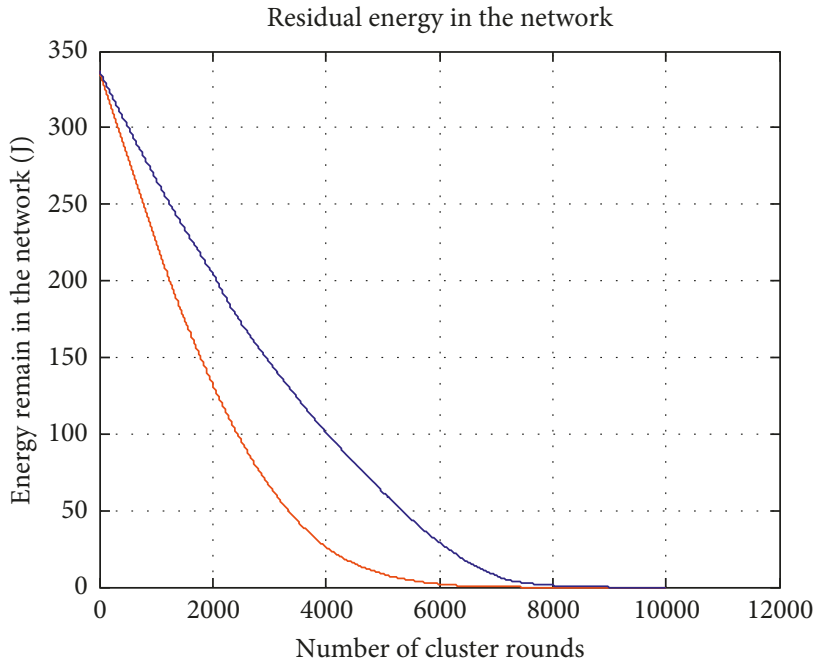

(c)

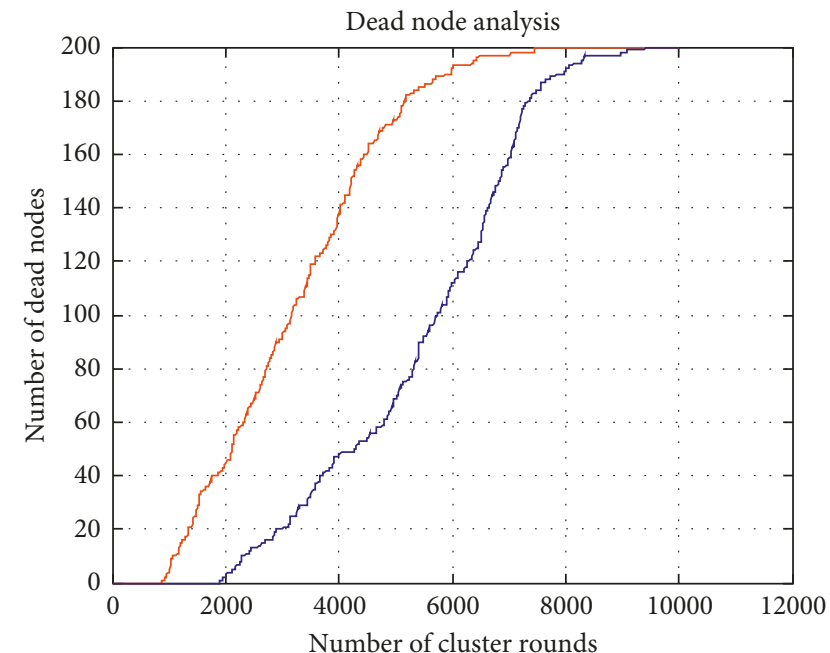

(b)

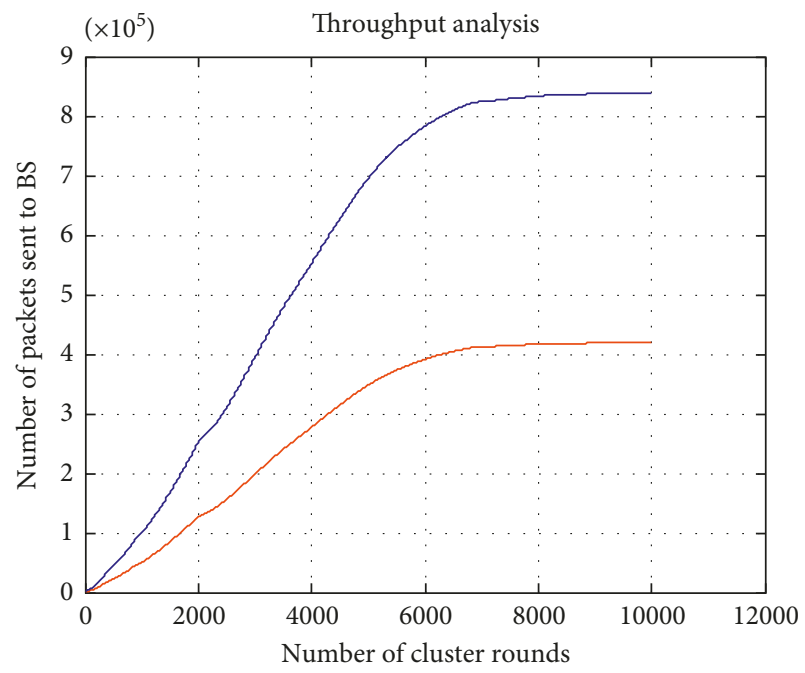

(d)

Figure 1: (a) Number of alive nodes versus number of cluster rounds. (b) Number of dead nodes versus number of cluster rounds. (c) Energy remaining in the network versus number of cluster rounds. (d) Number of data packets sent to BS versus number of cluster rounds.

BS. Hence, our protocol outperforms than the LEACH with this aspect in the form of stability period and lifetime presented in Figures 1(a) and 1(b). Colour used for presentation is blue for the proposed protocol and red colour is used for the former protocol. As per LEACH, there is less guarantee that the selected node is inside the network premises and not on the verge of vicinity or in the vicinity. Assumption of single hop and arbitrary cluster size decreases the energy efficiency of the 


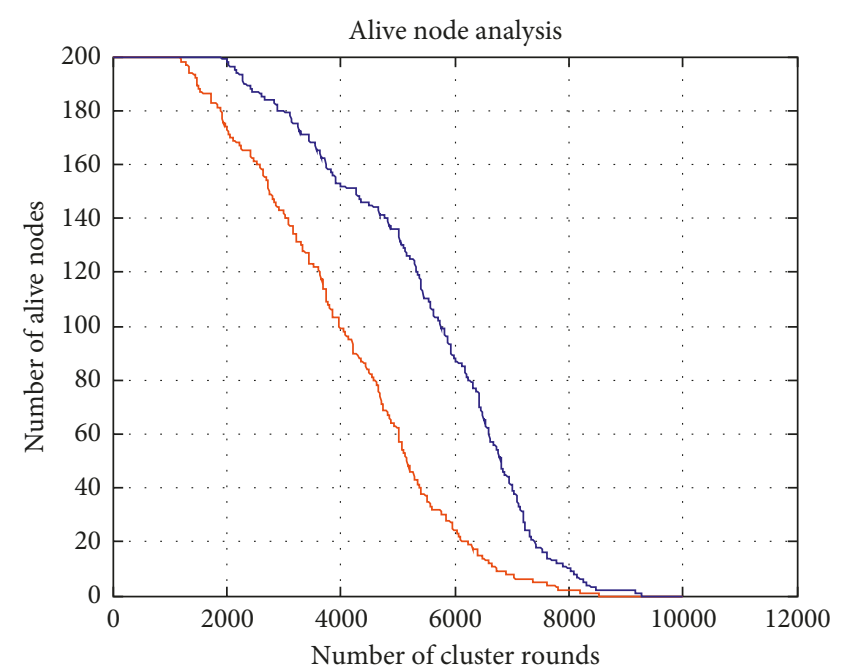

(a)

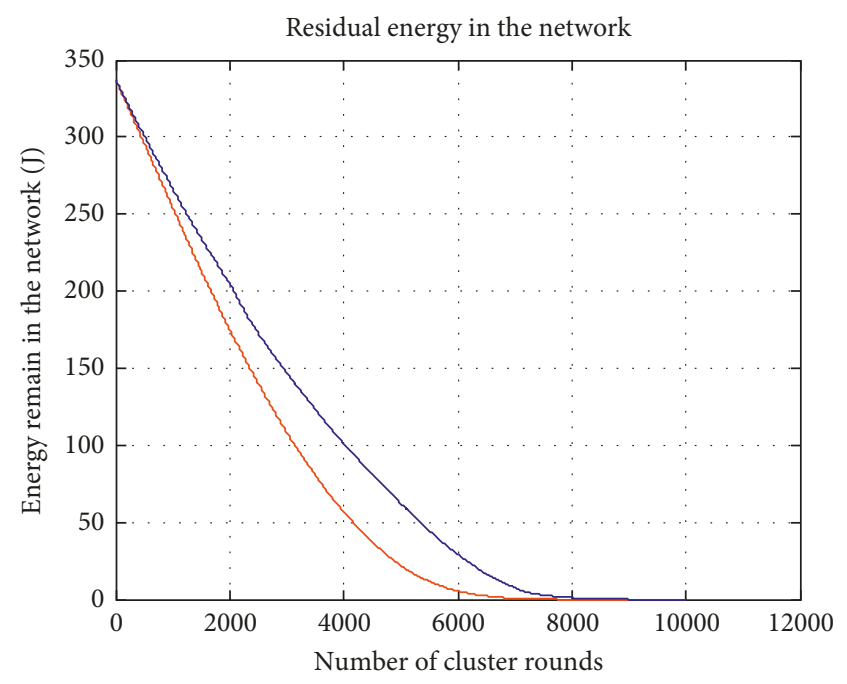

(c)

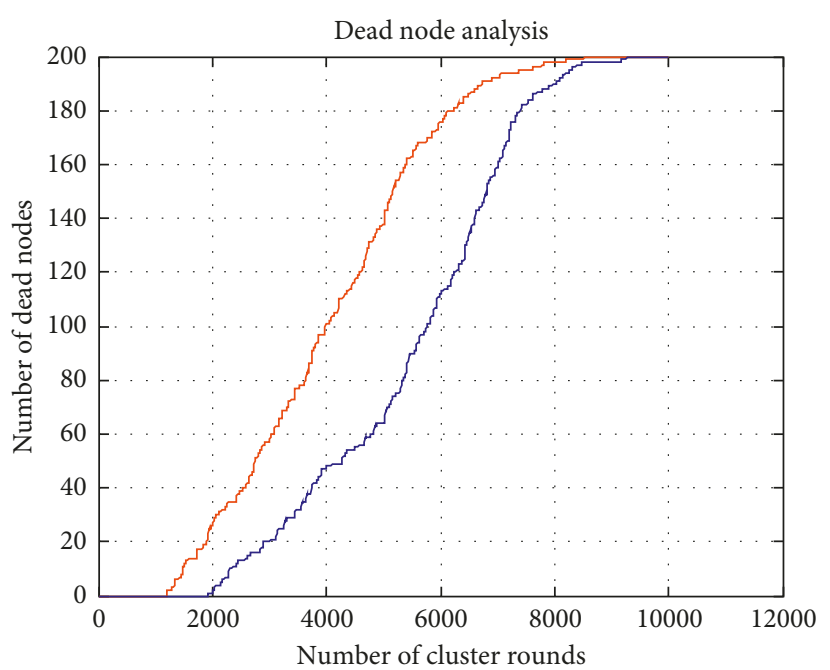

(b)

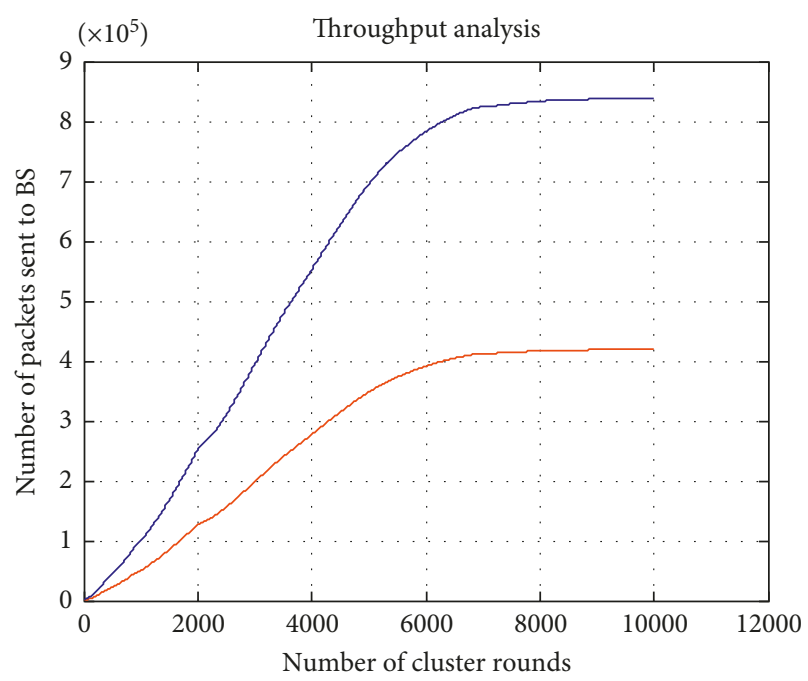

(d)

Figure 2: (a) Number of alive nodes versus number of cluster rounds. (b) Number of dead nodes versus number of cluster rounds. (c) Energy remaining in the network versus number of cluster rounds. (d) Number of data packets sent to BS versus number of cluster rounds.

network. The proposed protocol solves this issue with reduction in internal overhead packets, well assured selected $\mathrm{CH}$, and in the network premises. Hence, controlled energy utilization results in improved energy remaining in the network than LEACH protocol as shown in Figure 1(c). Finally, available energy is utilized efficiently and systematically for longer span of time improves throughput from the network shown in Figure 1(d).

6.2. Validation with SEP Protocol. As selection of $\mathrm{CH}$ is based on energy rather than the random selection of LEACH, it offers best suitable node to be $\mathrm{CH}$. In $\mathrm{LEACH}$, probability of node to be $\mathrm{CH}$ is equal for all nodes, but in SEP, probability of $\mathrm{CH}$ selection is different for two different nodes. Hence, it offers better performance than LEACH protocol in heterogeneous WSN. Basically SEP is proposed to introduce energy heterogeneity in WSN, that is, in the form of advanced nodes other than normal node as per [14]. But in the SEP protocol, there are two levels of probability function to differentiate between advanced and normal node for the role of $\mathrm{CH}$. This varies energy level excursion and hence energy remain in the network fluctuate greatly. Finally, depleting the energy faster results in reduced performance parameters of the network. It normally punishes the high energy node and results in reduction of stability period and lifetime. As shown in Figure 2(a), lifetime Figure 2(b) and energy remaining in the network Figure 2(c) are compared with the proposed protocol. The graph is presented with two colours as red is used for former protocol and blue is used for proposed protocol. As the proposed protocol is free from the varying probability function and changing energy level utilization, it improves energy remaining in the network or residual energy in the network and enhances the stability period, lifetime, and hence number of data packets sent towards base station in the form of Figure 2(d). 


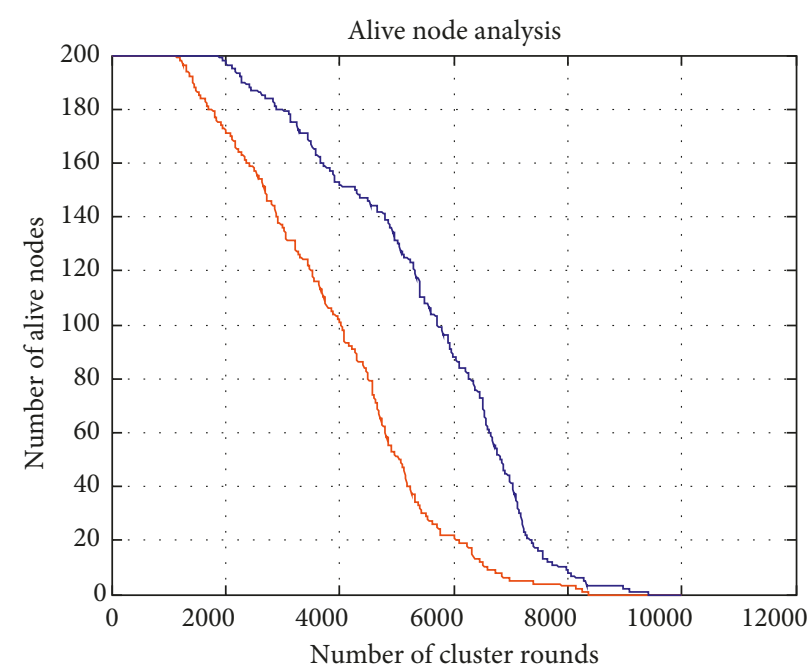

(a)

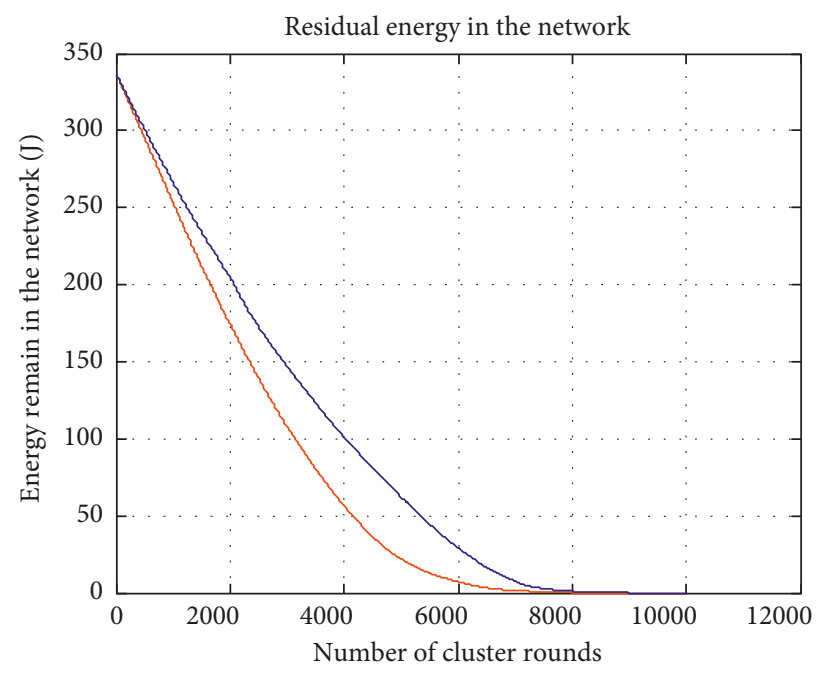

(c)

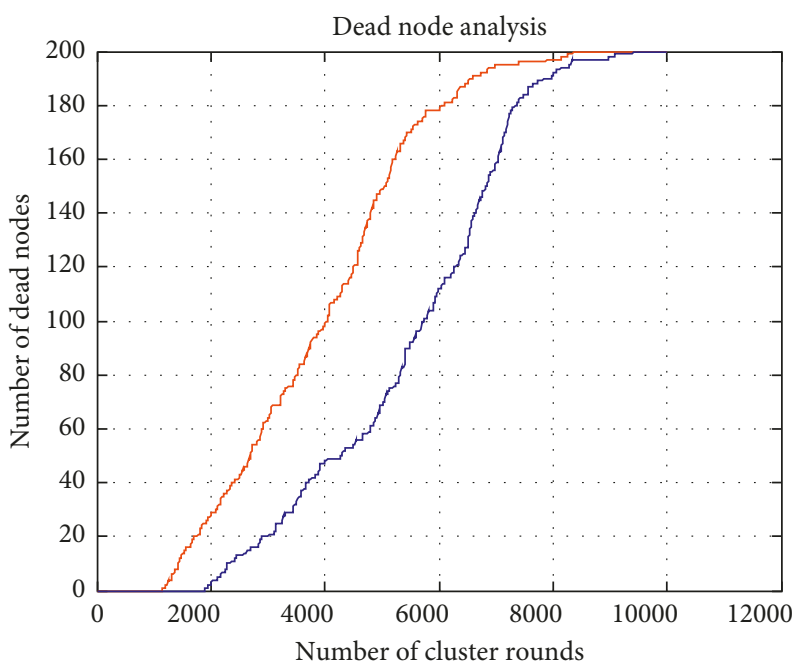

(b)

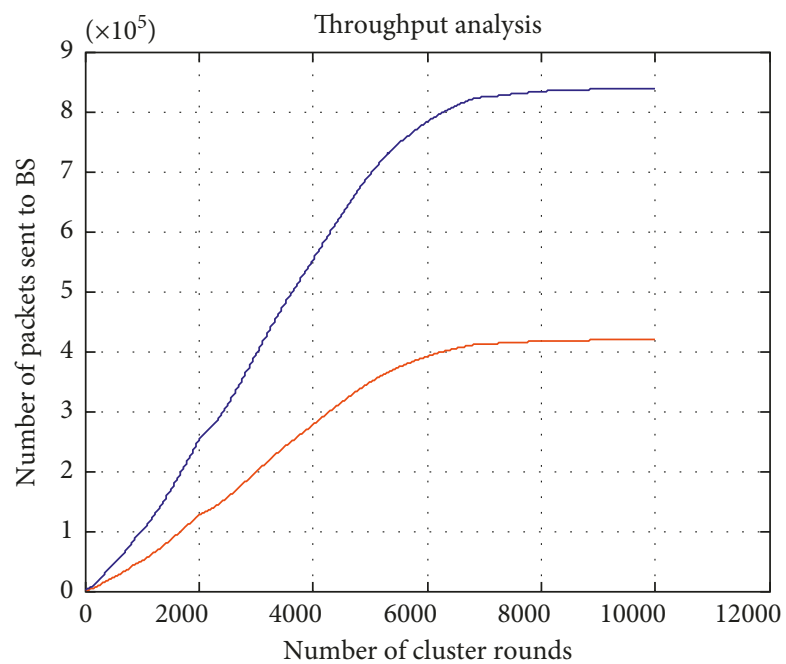

(d)

Figure 3: (a) Number of alive nodes versus number of cluster rounds. (b) Number of dead nodes versus number of cluster rounds. (c) Energy remaining in the network versus number of cluster rounds. (d) Number of data packets sent to BS versus number of cluster rounds.

TABLe 2: Performance validation table.

\begin{tabular}{|c|c|c|c|c|c|c|c|c|}
\hline \multirow{2}{*}{ Serial Number } & \multicolumn{5}{|c|}{ Name of the protocol to be simulated } & \multicolumn{3}{|c|}{$\%$ enhancement on } \\
\hline & Performance parameter & LEACH & SEP & DEEC & Proposed & LEACH & SEP & DEEC \\
\hline 1 & First node death (stability) & 874 & 1206 & 1136 & 1931 & 221 & 160 & 170 \\
\hline 2 & Tenth node death & 1345 & 1903 & 1710 & 2896 & 215 & 152 & 170 \\
\hline 3 & All nodes death (lifetime) & 7254 & 8199 & 8375 & 9284 & 128 & 113 & 110 \\
\hline \multicolumn{6}{|c|}{ Overall performance parameter improvement with proposed protocol } & 188 & 141 & 150 \\
\hline
\end{tabular}

6.3. Validation with DEEC Protocol. DEEC protocol proposed by author with the approach of average energy in the network and initial lifetime calculations in the form of number of round are given in [15]. In this protocol, global knowledge of the network needs to be updated, and probability threshold function also plays an important role for $\mathrm{CH}$ selection. As there is variation in energy level during real-time operation inside the network, computing the average energy reference for the $\mathrm{CH}$ selection for subsequent cluster round calculation is affected by energy balancing inside the network. One more point needed to be highlighted is that different probability functions incorporate inside the protocol, which has bad effects on energy remain in the network. Hence, reduce the lifetime of sensor network as depicted in Figures 3(a) and 3(b) in the form of blue and red colour. Energy unbalancing of DEEC is depicted in the form of energy remaining in the network as in Figure 3(c). Throughput of the former protocol is also 
getting reduced, presented in Figure 3(d). However, the proposed protocol does not have any varying probability function, and the criteria for $\mathrm{CH}$ selection are deterministic. Hence, the proposed protocol outperforms than the former DEEC.

Some of the figures in Table 2 are recorded from the simulation based on the parameter set given in Table 1. Here, the first value represents stability that is nothing, but first sensor node death occurred during operation. Second value is for tenth dead node, which is the death of 10th working node from network field to estimate death rate with working protocol and to find maximum possible lifetime remain with network. Finally, Lifetime is the parameter which depicts the death of all deployed sensor nodes from the network. Table 2 shows statistics for validating the proposed protocol simulation based on performance parameters such as stability, death of tenth node, and lifetime in the form of cluster round, which depicts that the proposed design outperforms for HWSN, which is based on energy heterogeneity. Hence, the proposed clustering protocol supports load balancing, and also it improves stability and lifetime of network than the former well-known protocols.

\section{Conclusion}

In the proposed clustering protocol of HWSN, selection of $\mathrm{CH}$ is based on newly devised parameter in the form of node index depending on current available energy, initial energy, and hop count from the base station. The node selected for the role of temporary $\mathrm{CH}$ of each zone is situated off the vicinity and at a limited distance from BS. This approach assures available energy utilized for the sake of data manipulation and communication, it leads to improved energy remaining in the network and enhance the throughput from the network. Finally, the node which has a better value of NI than temporary selected $\mathrm{CH}$ and of the vicinity can be the final $\mathrm{CH}$. Other achievement with proposed approach is internal overheads getting reduced to greater extent with the initial division of network in sections. Due to this, formed clusters have controlled size than arbitrary size cluster of the earlier protocols. All these approaches contribute to improve energy efficiency; force to enhance stability period and lifetime; and improve residual energy of the network and hence throughput than existing protocol.

We try to explore our proposed work with a different mechanism as spanning tree for route finding, try to use the mobile sink scheme to enhance the performance of the proposed design and finally try to use weight calculation method based on different parameters associated with sensor node for finding the best possible node as the $\mathrm{CH}$ for the role of $\mathrm{CH}$.

\section{Conflicts of Interest}

The authors declare that they have no conflicts of interest.

\section{References}

[1] A. A. Abbasi and M. Younis, "A survey on clustering algorithms for wireless sensor networks," Computer Communications, vol. 30, no. 14-15, pp. 2826-2841, 2007.
[2] S. V. Purkar and R. S. Deshpande, "A review on energy efficient clustering protocols of heterogeneous wireless sensor network," International Journal of Engineering and Technology, vol. 9, no. 3, pp. 2514-2527, 2017.

[3] S. A. Nikolidakis, D. Kandris, D. D. Vergados, and C. Douligeris, "Energy efficient routing in wireless sensor networks through balanced clustering," Algorithms, vol. 6, no. 1, pp. 29-42, 2013.

[4] G. Abdul-Salaam, A. H. Abdullah, M. H. Anisi, A. Gani, and A. Alelaiwi, "A comparative analysis of energy conservation approaches in hybrid wireless sensor networks data collection protocols," Telecommunication Systems, vol. 16, no. 1, pp. 159-179, 2016.

[5] S. Singh, A. Malik, and R. Kumar, "Energy efficient heterogeneous DEEC protocol for enhancing lifetime in WSNs," Engineering Science and Technology an International Journal, vol. 20, no. 1, pp. 345-353, 2017.

[6] A. Kashaf, N. Javaid, Z. A. Khan, and I. A. Khan, "TSEP: threshold-sensitive stable election protocol for WSNs," in Proceedings of the 10th International Conference on Frontiers of Information Technology, pp. 164-168, Islamabad, Pakistan, December 2012.

[7] P. Saini and A. K. Sharma, "E-DEEC-enhanced distributed energy efficient clustering scheme for heterogeneous WSN," in Proceedings of the 1st International Conference on Parallel, Distributed and Grid Computing (PDGC 2010), pp. 205-210, Solan, India, October 2010.

[8] B. Manzoor, N. Javaid, O. Rehman et al., "Q-LEACH: a new routing protocol for WSNs," in Proceedings of the International Workshop on Body Area Sensor Networks (BASNet-2013), SciVerse Science Direct 2013, vol. 19, pp. 926-931, Halifax, NS, Canada, June 2013.

[9] K. Rana and M. Zaveri, "Synthesized cluster head selection and routing for two tier wireless sensor network," Journal of Computer Network and Communications, vol. 2013, Article ID 578241, 11 pages, 2013.

[10] W. R. Heinzelman, A. Chandrakasan, and H. Balakrishnan, "Energy-efficient communication protocol for wireless microsensor networks," in Proceedings of the Hawaii International Conference on System Sciences, pp. 1-10, Maui, HI, USA, January 2000.

[11] M. Y. Wang, J. Ding, W. Chen, and W. Guan, "SEARCH: a stochastic election approach for heterogeneous wireless sensor networks," IEEE Communications letters, vol. 19, no. 3, pp. 443-446, 2015.

[12] H. Li and J. Liu, "Double cluster based energy efficient routing protocol for wireless sensor network," International Journal of Wireless Information Networks, vol. 23, no. 1, pp. 40-48, 2016.

[13] O. Younis and S. Fahmy, "HEED: a hybrid, energy-efficient, distributed clustering approach for ad-hoc sensor networks," IEEE Transactions on Mobile Computing, vol. 3, pp. 366-379, 2004.

[14] G. Smaragdakis, I. Matta, and A. Bestavros, "SEP: a stable election protocol for clustered heterogeneous wireless sensor network," in Proceedings of the Second International Workshop on Sensor and Actuator Network Protocols and Applications (SANPA'04), pp. 1-6, Boston, MA, USA, August 2004.

[15] L. Qing, Q. Zhu, and M. Wang, "Design of a distributed energy-efficient clustering algorithm for heterogeneous wireless sensor network," Computer Communications, vol. 29, no. 12, pp. 2230-2237, 2006.

[16] B. Elbhiri, R. Saadane, S. E. Fkihi, and D. Aboutajdine, "Developed distributed energy-efficient clustering (DDEEC) for 
heterogeneous wireless sensor networks," in Proceedings of the 5th International Symposium on $I / V$ Communications and Mobile Network (ISVC), pp. 1-4, Rabat, Morocco, SeptemberOctober 2010.

[17] N. Javaid, M. B. Rasheed, M. Imran et al., "An energy-efficient distributed clustering algorithm for heterogeneous WSNs," EURASIP Journal on Wireless Communications and Networking, Springer, vol. 2015, p. 151, 2015.

[18] D. Kumar, T. C. Aseri, and R. B. Patel, "EEHC: energy efficient heterogeneous cluster scheme for wireless sensor networks," Computer Communication, vol. 32, no. 4, pp. 662-667, 2009.

[19] J. Lloret, M. Garcia, D. Bri, and J. R. Diaz, "A cluster-based architecture to structure the topology of wireless sensor networks," Sensors, vol. 9, no. 12, pp. 10513-10544, 2009.

[20] M. Faheem, M. Z. Abbas, G. Tuna, and V. C. Gungor, "EDHRP: energy efficient event driven hybrid routing protocol for densely deployed wireless sensor networks," Journal of Network and Computer Applications, vol. 58, pp. 309-326, 2015.

[21] S. Chand, S. Singh, and B. Kumar, "Heterogeneous HEED protocol for wireless sensor networks," Wireless Personal Communications, vol. 77, no. 3, pp. 2117-2139, 2014. 


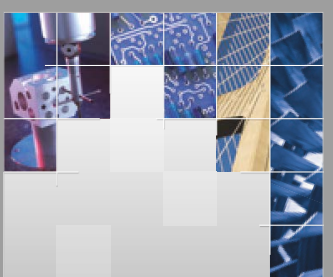

\section{Enfincering}
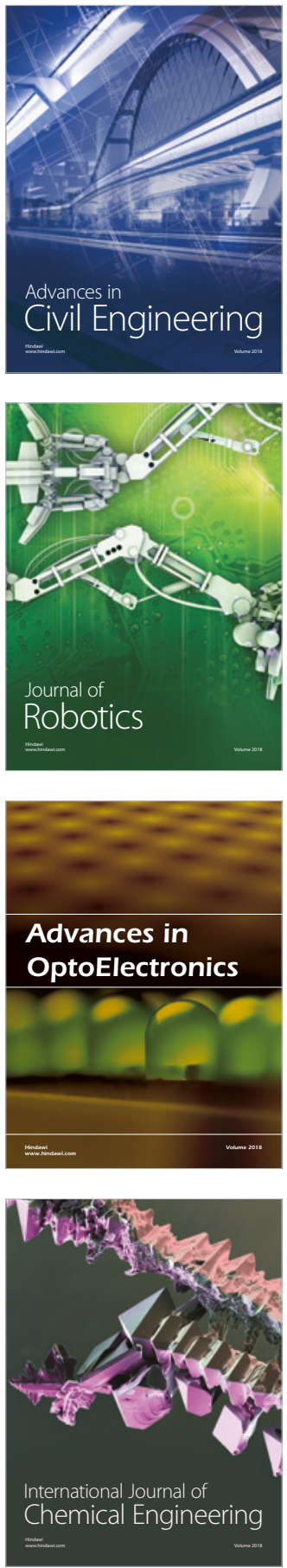

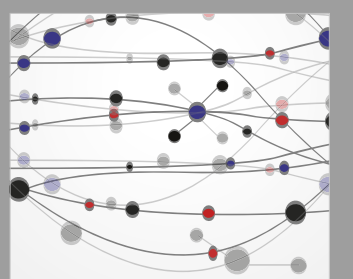

\section{Rotating \\ Machinery}

The Scientific World Journal

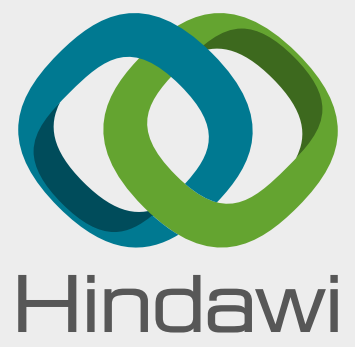

Submit your manuscripts at

www.hindawi.com
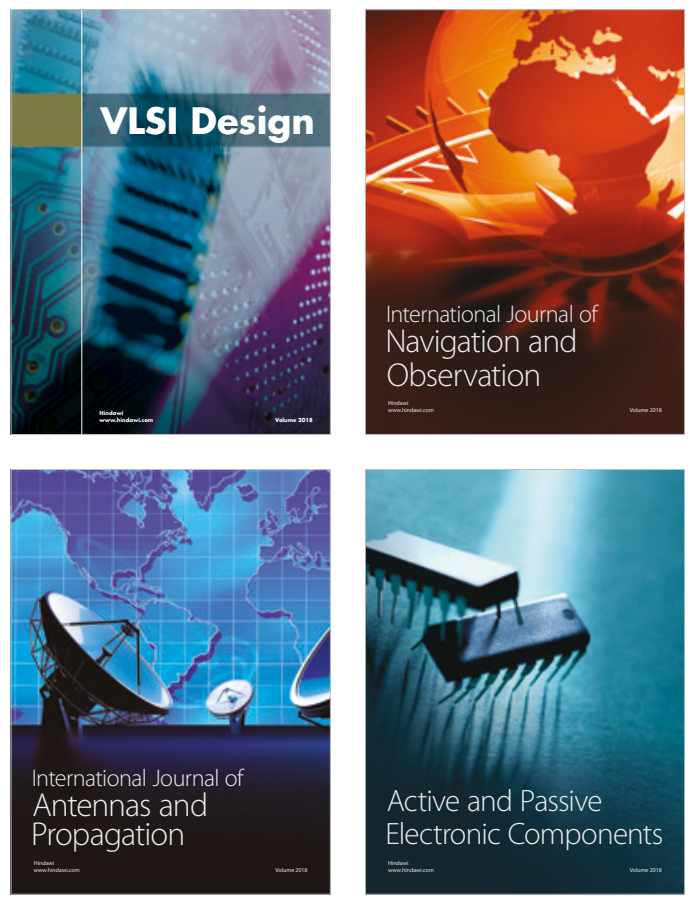
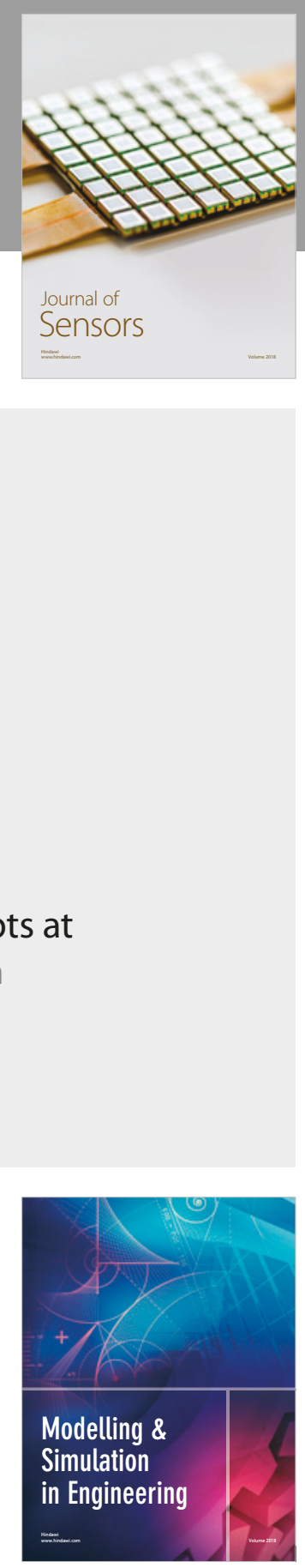

\section{Advances \\ Multimedia}
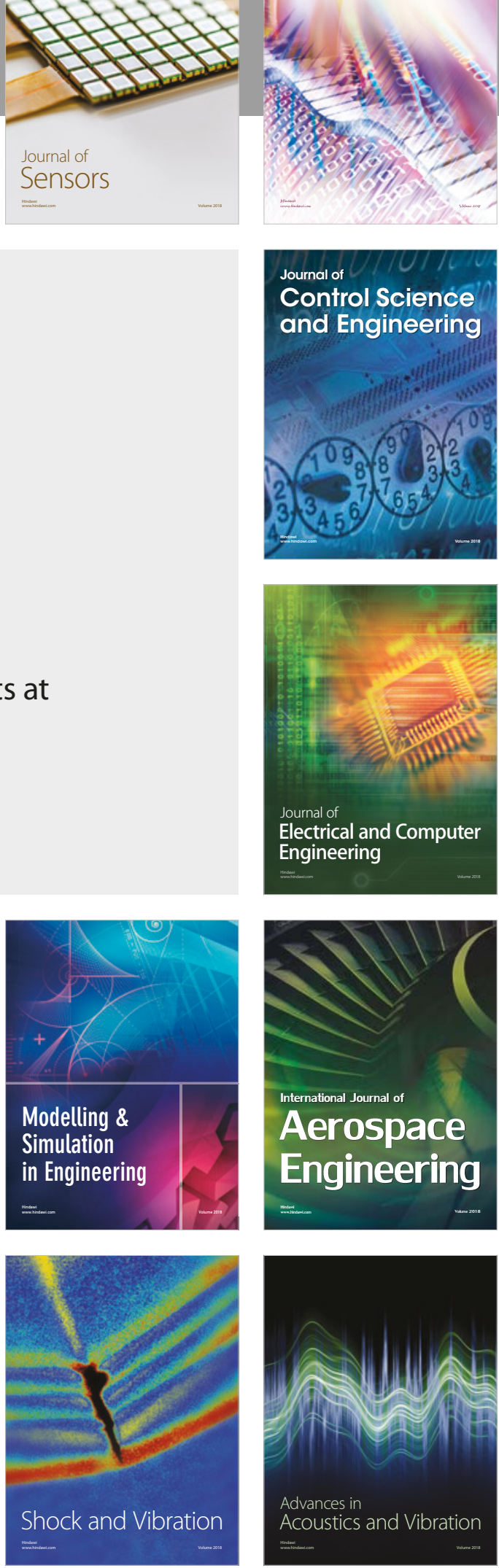